\title{
Analytic Subordination Consequences of Free Markovianity
}

\author{
Dan Voiculescuf \\ Department of Mathematics \\ University of California \\ Berkeley, California 94720-3840
}

\section{Introduction}

In [4], under some easy to remove genericity assumptions, we proved the analytic subordination of the Cauchy transforms of distributions $G_{\mu_{X+Y}}$ and $G_{\mu_{X}}$ for a pair of freely independent self-adjoint random variables $X, Y$. We used this to obtain inequalities among $p$-norms of densities of distributions, free entropies and Riesz energies. This was followed by P.Biane's discovery [1] that subordination extends, roughly speaking, to the resolvents, i.e. is an operator-valued occurrence. He also showed that this implies a noncommutative Markovtransitions property for free increment processes and that there are similar subordination results for multiplicative processes.

The analytic function approach in [4] and the combinatorial one in [1] did not shed much light on why analytic subordination appears in this context. In [6] we found a simple explanation of this phenomenon based on the coalgebra structure associated with the free difference quotient derivation. In the additive case this also led to a far reaching generalization of the analytic subordination result to the $B$-valued case, i.e. to free independence with amalgamation over an algebra $B$.

Here, based on simple operator-valued analytic function considerations, we build further on the result in [6]. We derive more general analytic subordination results for a freely Markovian triple $A, B, C$. This also includes the $B$-valued extension of the result for multiplication of free unitary variables.

\footnotetext{
${ }^{1}$ Supported in part by National Science Foundation grant NSF-9500308.
} 


\section{Notation and Preliminaries}

Throughout, $(M, \tau)$ will denote a tracial $W^{*}$-probability space, i.e. a von Neumann algebra endowed with a normal faithful trace state. A triple of von Neumann subalgebras $A, B, C$ contained in $M$ and containing $\mathbb{C} 1$ is freely Markovian (see [5] or [7]) if $A$ and $C$ are $B$-free in $\left(M, E_{B}\right)$ where $E_{B}$ is the canonical conditional expectation of $M$ onto $B$ (see [7] or [8]). If $A, B$ are von Neumann subalgebras of $M$ containing 1, we shall denote by $A \vee B$ or $W^{*}(A \cup B)$ the von Neumann subalgebra generated by $A \cup B$. If $X=X^{*} \in M$ and $1 \in B \subset M$ is a $*$-subalgebra we shall denote by $B[X]$ the $*$-subalgebra generated by $B$ and $X$ and we shall denote by $E_{B[X]}$ the conditional expectation onto the von Neumann subalgebra $W^{*}(B[X])$. For several selfadjoint elements $X, Y, Z, \ldots$ (noncommuting) we shall also use notations $B[X, Y, Z], E_{B[X, Y, Z]}$, etc.

If $A$ is a unital $C^{*}$-algebra we denote by $\mathbb{H}_{+}(A)=\{T \in A \mid \operatorname{Im} T \geq \varepsilon 1$ for some $\varepsilon>0\}$ the upper half-plane of $A$ and by $\mathbb{H}_{-}(A)=-\mathbb{H}_{+}(A)$ the lower half-plane. Note that $T \in \mathbb{H}_{+}(A) \Leftrightarrow T^{-1} \in \mathbb{H}_{-}(A)$, in particular elements in $\mathbb{H}_{ \pm}(A)$ are invertible. Also, $A_{s a}$ will denote the selfadjoint elements in $A$.

The open unit ball will be denoted $\mathbb{D}(A)=\{T \in A \mid\|T\|<1\}$. For balls of radius $R$ we shall also use the notations $\mathbb{D}_{R}(A)=R \mathbb{D}(A)$.

\section{Analytic Subordination}

We begin by recalling the result ([6] Theorem 3.8) which serves as our starting point.

3.1 Theorem. Let $1 \in B \subset M$ be a $W^{*}$-subalgebra and let $X=X^{*} \in M, Y=Y^{*} \in M$. Assume $X, Y$ are $B$-free in $\left(M, E_{B}\right)$. Then there is a holomorphic map $F: \mathbb{H}_{+}(B) \rightarrow \mathbb{H}_{+}(B)$ such that

$$
E_{B[X]}((X+Y)-b)^{-1}=(X-F(b))^{-1}
$$

if $b \in \mathbb{H}_{+}(B)$.

3.2 Proposition. Let $1 \in B \subset M$ and $1 \in A \subset M$ be $W^{*}$-subalgebras and let $X=X^{*} \in M$. Assume $A$ and $X$ are $B$-free in $\left(M, E_{B}\right)$. Then there is a holomorphic map $F: \mathbb{H}_{+}(A) \rightarrow \mathbb{H}_{+}(B)$ such that

$$
E_{B[X]}(a-X)^{-1}=(F(a)-X)^{-1}
$$


Proof. It is easily seen that

$$
F_{1}(a)=\left(E_{B[X]}(a-X)^{-1}\right)^{-1}+X
$$

is a well-defined holomorphic map

$$
\mathbb{H}_{+}(A) \rightarrow \mathbb{H}_{+}\left(W^{*}(B[X])\right) .
$$

We must show that $F_{1}\left(\mathbb{H}_{+}(A)\right) \subset B$. By Theorem 3.1,

$$
F_{1}\left(i \varepsilon I+A_{s a}\right) \subset \mathbb{H}_{+}(B)
$$

if $\varepsilon>0$. Indeed, if $-Y \in A_{s a}$ then Proposition 3.2 gives

$$
E_{B[X]}(i \varepsilon I-Y-X)^{-1}=(b-X)^{-1}
$$

for some $b \in \mathbb{H}_{+}(B)$ which means $F_{1}(i \varepsilon I-Y)=b$.

Since $\varepsilon I+A_{s a}$ is a uniqueness set for holomorphic functions on $\mathbb{H}_{+}(A)$, the inclusion $F_{1}\left(i \varepsilon I+A_{s a}\right) \subset B$ implies $F_{1}\left(\mathbb{H}_{+}(A)\right) \subset B$ (use functionals $f \in M_{*}, f \mid B=0$ to transform the given inclusion into $\left.\left(f \circ F_{1}\right) \mid i \varepsilon I+A_{s a}=0\right)$.

3.3 Proposition. Let $A, B, C$ be a freely Markovian triple of $W^{*}$-subalgebras in $(M, \tau)$. Then there is a holomorphic map

$$
F: \mathbb{H}_{+}(A) \times \mathbb{H}_{+}(C) \rightarrow B
$$

such that

$$
\left(E_{A \vee B}(a+c)^{-1}\right)^{-1}=a+F(a, c)
$$

if $a \in \mathbb{H}_{+}(A), \quad c \in \mathbb{H}_{+}(C)$.

Proof. Clearly, we have $a+c \in \mathbb{H}_{+}(M), \quad(a+c)^{-1} \in \mathbb{H}_{-}(M), \quad E_{A \vee B}(a+c)^{-1} \in \mathbb{H}_{-}(M)$,

$$
\left(E_{A \vee B}(a+c)^{-1}\right)^{-1} \in \mathbb{H}_{+}(M) .
$$

Hence

$$
F_{1}: \mathbb{H}_{+}(A) \times \mathbb{H}_{+}(C) \rightarrow A \vee B \vee C
$$

given by

$$
F_{1}(a, c)=\left(E_{A \vee B}(a+c)^{-1}\right)^{-1}-a
$$


is well-defined and holomorphic. Thus, the proof reduces to showing that $F_{1}\left(\mathbb{H}_{+}(A) \times \mathbb{H}_{+}(C)\right) \subset B$. Since $\left(i \varepsilon I+A_{s a}\right) \times\left(i \varepsilon I+C_{s a}\right)$, where $\varepsilon>0$ is a set of uniqueness for holomorphic maps on $\mathbb{H}_{+}(A) \times \mathbb{H}_{+}(C)$, it suffices to show that

$$
\left(E_{A \vee B}((X+Y)+2 \varepsilon i)^{-1}\right)^{-1}-X \in B
$$

when $X \in A_{s a}, \quad Y \in C_{s a}$. Since $(X+Y+2 \varepsilon i)^{-1} \in W^{*}(B[X]) \vee C$ and $A$ and $C$ are $B$-free in $\left(M, E_{B}\right)$ we have

$$
E_{A \vee B}(X+Y+2 \varepsilon i)^{-1}=E_{B[X]}(X+Y+2 \varepsilon i)^{-1} .
$$

By Theorem 3.1 we have

$$
E_{B[X]}(X+Y+2 \varepsilon i)^{-1}=(X+b)^{-1}
$$

for some $b \in \mathbb{H}_{+}(B)$. In particular

$$
\left(E_{A \vee B}(X+Y+2 \varepsilon i)^{-1}\right)^{-1}-X \in B .
$$

3.4 Lemma. If $x \in A$, where $A$ is a unital $C^{*}$-algebra, the following are equivalent:

(i) $\|x\|<1$

(ii) $1-x$ is invertible and $2 \operatorname{Re}(1-x)^{-1} \geq(1+\varepsilon)$ for some $\varepsilon>0$.

Proof. This fact, not new, is for instance an immediate consequence of unitary dilation [3] and of the corresponding fact when $A=\mathbb{C}$. For the reader's convenience, here is a direct proof.

Both (i) and (ii) imply $1-x$ is invertible, in which case we have:

$$
(1-x)^{-1}+\left(1-x^{*}\right)^{-1}=1+(1-x)^{-1}\left(1-x x^{*}\right)^{-1}\left(1-x^{*}\right)^{-1} .
$$

To prove (i) $\Rightarrow$ (ii) remark that $1-x x^{*} \geq\left(1-\|x\|^{2}\right) 1$ and that

$$
\left\|\left(1-x^{*}\right)^{-1} \xi\right\| \geq\left\|1-x^{*}\right\|^{-1}\|\xi\| \geq 2^{-1}\|\xi\|
$$

when $A$ acts on a Hilbert space $\mathcal{H}$ and $\xi \in \mathcal{H}$. This gives $(1-x)^{-1}\left(1-x x^{*}\right)\left(1-x^{*}\right)^{-1} \geq$ $4^{-1}\left(1-\|x\|^{2}\right) 1$.

To show (ii) $\Rightarrow$ (i) note that (ii) implies $(1-x)^{-1}\left(1-x x^{*}\right)\left(1-x^{*}\right)^{-1} \geq \varepsilon 1$ so that $1-x x^{*} \geq 0$ and $1-x x^{*}$ is invertible, which gives (i). 
3.5 Proposition. Let $A, B, C$ be a freely Markovian triple of $W^{*}$-algebras in $(M, \tau)$ and let $\Omega=\left\{(a, c) \in A \times C \mid a\right.$ invertible, $\left.\left\|a^{-1} c\right\|<1\right\}$. Then there is a holomorphic map $\Phi: \Omega \rightarrow B$ such that if $(a, c) \in \Omega$, then $\left\|a^{-1} \Phi(a, c)\right\|<1$ and $E_{A \vee B}(a-c)^{-1}=(a-\Phi(a, c))^{-1}$.

Proof. Let $(a, c) \in \Omega$. Then $a-c$ is invertible, the inverse being $\left(1-a^{-1} c\right)^{-1} a^{-1}$. Using Lemma 3.41 we have

$$
E_{A \vee B}\left(1-a^{-1} c\right)^{-1} \in \frac{1}{2}-i \mathbb{H}_{+}(M)
$$

and hence

$$
E_{A \vee B}\left(1-a^{-1} c\right)^{-1}=(1-x)^{-1}
$$

for some $x \in \mathbb{D}(M)$. Moreover there is a holomorphic map $\Psi: \Omega \rightarrow \mathbb{D}(M)$ such that $\Psi(a, c)=1-\left(E_{A \vee B}\left(1-a^{-1} c\right)^{-1}\right)^{-1}$.

Let $\Phi_{1}: \Omega \rightarrow M$ be defined by $\Phi_{1}(a, c)=a \Psi(a, c)$. Clearly $\Phi_{1}$ is holomorphic, $\left\|a^{-1} \Phi_{1}(a, c)\right\|<1$ and

$$
E_{A \vee B}(a-c)^{-1}=\left(E_{A \vee B}\left(1-a^{-1} c\right)^{-1}\right) a^{-1}=(1-\Psi(a, c))^{-1} a^{-1}=\left(a-\Phi_{1}(a, c)\right)^{-1} .
$$

Thus the proof reduces to showing that $\Phi_{1}(a, c) \in B$.

The open set $\Omega$ is connected. Indeed, if $(a, c) \in \Omega$ then $(\lambda a, c) \in \Omega$ for all $\lambda \in \mathbb{C}$, $|\lambda| \geq 1$. In particular there is a segment in $\Omega$ connecting $(a, c)$ and $(N a, c)$ where $N \geq 1$ is such that $\left\|(N a)^{-1}\right\| \leq(1+\|c\|)^{-1}$. Then $(N a, t c+(1-t) 1) \in \Omega$ for $0 \leq t \leq 1$ and we have a path from $(a, c)$ to $(N a, 1)$. If $N a=u(N|a|)$ is the polar decomposition, then $N|a| \geq(1+\varepsilon) 1$ for some $\varepsilon>0$ and the elements $(u((1-t) N|a|+t(1+\varepsilon) 1,1), 0 \leq t \leq 1$, are in $\Omega$ connecting $(N a, 1)$ and $((1+\varepsilon) u, 1)$. Finally $u=\exp (i h)$ for some $h=h^{*} \in A$ and $((1+\varepsilon) \exp (i t h), 1) \in \Omega$ for $0 \leq t \leq 1$ continue our path to $((1+\varepsilon) 1,1)$, etc.

Since $\Omega$ is connected, the set

$$
\omega=\left\{(a, c) \in A \times C \mid\|a-3 i 1\|<1, \operatorname{Im} c \leq-\frac{1}{2} 1,\|c\|<1\right\} \subset \mathbb{H}_{+}(A) \times \mathbb{H}_{-}(C)
$$

and $\omega \subset \Omega$ is a uniqueness set for holomorphic functions in $\Omega$. Moreover, using Proposition 3.3 we have $\Phi(a, c)=F(a,-c) \in B$ where $(a, c) \in \omega$ and $F$ is the function in Proposition 3.3 .

3.6 Theorem. Let $1 \in B, 1 \in C$ be $W^{*}$-algebras in $(M, \tau)$ and let $u$ be a unitary element in $M$. Assume $C$ and $\left\{u, u^{*}\right\}$ are $B$-free in $\left(M, E_{B}\right)$. Then there is a holomorphic map

$$
G: \mathbb{D}(C) \rightarrow \mathbb{D}(B)
$$


such that

$$
E_{B\left[u, u^{-1}\right]}(u-c)^{-1}=(u-G(c))^{-1}
$$

if $c \in \mathbb{D}(B)$.

Proof. This follows immediately from Proposition 3.5 since $(u, c) \in \Omega$ if $c \in \mathbb{D}(C)$ and $\left\|u^{-1} \Phi(u, c)\right\|<1 \Leftrightarrow\|\Phi(u, c)\|<1$ and we take $G(c)=\Phi(u, c)$.

\section{References}

[1] P.Biane, Processes with free increments, Math. Z. 227 (1998), 143-174.

[2] E.Hille and R.S.Phillips, Functional Analysis and Semigroups, vol. 31 American Math Society Colloqu. Publ., Rhode Island, 1957.

[3] B.Sz.-Nagy and C.Foias, Analyse Harmonique des Opérateurs de l'espace de Hilbert, Masson et Cie, Paris, 1967.

[4] D.Voiculescu, D. Voiculescu, The analogues of entropy and of Fisher's information measure in free probability theory, I. Commun. Math. Phys. 155 (1993), 71-92.

[5] D.Voiculescu, The analogues of entropy and of Fisher's information measure in free probability theory, VI: Liberation and mutual free information. Adv. Math. 146 (1999), 101-106.

[6] D.Voiculescu, The coalgebra of the free difference quotient and free probability. International Math. Res. Notices No. 2 (2000), 79-106.

[7] D.Voiculescu, Lectures on Free Probability Theory, in Lectures on Probability Theory and Statistics, Saint-Flour XXVIII (1998) Lecture Notes in Math. vol. 1738, 279-349.

[8] D.Voiculescu, K.Dykema and A.Nica, Free Random Variables, CRM Monograph Series vol. I, American Math Society, Rhode Island, 1992. 